\title{
Interacting Streamers in Air: The Evolution of the Space-Charge Layer in Their Heads
}

\author{
Alejandro Luque and Ute Ebert
}

\begin{abstract}
Three-dimensional plots of the evolution of the space-charge layers of two interacting negative streamers show how the electrostatic repulsion between the two heads affects the charge distribution in the heads and drives them apart.
\end{abstract}

Index Terms-Corona discharge, photoionization, streamers.

$\mathbf{T}$ HE THEORETICAL investigation of streamers-thin plasma channels that propagate through a nonionized gas suddenly exposed to high electric fields [1]—until very recently, has concentrated on single streamers only. But very often, streamers are observed in bunches propagating close together and in similar directions, either because they have branched from a single source or because the geometry and initial conditions favor the formation of multiple channels. Arrays of streamers are, e.g., created in the laboratory by means of multiple electrode needles [2], [3]. In addition, in sprite discharges above thunderclouds, one can observe multiple streamers [4], [5], where the interaction between them is probably the reason behind the "carrot-shape" of sprites.

In [6], it was shown that 2-D streamers, arranged in a periodic array, converge toward a uniformly translating state with the shape of the well-known selected Saffman-Taylor finger. In [7], a first study of the physics of interacting streamers in three dimensions was presented. It was shown that there exist the following two competing mechanisms: electrostatic repulsion of their heads and photoionization in the region between them, which tends to make them merge. In this paper, we illustrate these results with figures showing the geometrical aspects of the streamer evolution, in particular, the shape of the space-charge layer in full three dimensions.

The streamer model for oxygen-nitrogen mixtures that we use is detailed, e.g., in [7] and [8]. A major challenge in studying 3-D streamer dynamics is the high computational power required. In order to perform accurate simulations in present computers and manageable times, several numerical advances were needed. First of all, a proper refinement scheme that allows us to finely resolve the thin concentrated space-charge layer while not wasting time on the much coarser structures of

Manuscript received November 30, 2007; revised March 20, 2008. The work of A. Luque was supported by the Dutch STW, which is part of the Netherlands Organization for Scientific Research NWO, under Project 06501.

A. Luque is with Center of Mathematics and Computer Science (CWI), 1090 GB Amsterdam, The Netherlands (e-mail: A.Luque@cwi.nl).

U. Ebert is with the Center of Mathematics and Computer Science (CWI), 1090 GB Amsterdam, The Netherlands, and also with the Technical University of Eindhoven, 5600 MB Eindhoven, The Netherlands.

Digital Object Identifier 10.1109/TPS.2008.925706 the rest of the streamer [9], [10]. Then, a method had to be devised for a fast parallel solution of the Poisson equation in order to benefit from multiprocessor architectures [7]. Finally, an efficient method to calculate the photoionization source term, as described in [8], was applied.

At constant oxygen concentration, the typical length scales of the model are inversely proportional to the gas pressure; hence, it is convenient to use reduced lengths $p r$. The model, however, does not scale exactly with pressure, since collisional quenching of excited nitrogen molecules reduces the emission of photoionizing radiation. Thus, for higher pressures, the effects of photoionization on the streamer dynamics become smaller. Note however that the degree of photoionization was enough to suppress branching in all our simulations.

The simulations were started with two identical neutral seeds in air, under an electric field of $80\left(p_{0} / p\right) \mathrm{kV} \cdot \mathrm{cm}^{-1}$, where $p_{0}=1$ bar (see [7] for details). We studied negative streamers in air at 1 and 50 bar. Fig. 1 shows the electron density and electric field in the plane that intersects both streamers at their centers. The temporal evolution of the space-charge layer is shown in Figs. 2 and 3. These images where produced by extracting from the charge density the surface defined by $n_{e}(\mathbf{r})-n_{i}(\mathbf{r})=\max \left(n_{e}-n_{i}\right) / 2$.

The figures show the interplay between the following two relevant phenomena in streamer interaction: electrostatic repulsion and attraction through photoionization. In Fig. 2, the streamers coalesce after some evolution, despite the electrostatic repulsion between their heads, which is particularly visible, as shown in Fig. 2(c). Fig. 3, on the other hand, shows an evolution at a higher pressure. In that situation, there is not enough ionization in the space between the streamers to make them merge; therefore, they diverge. As shown in Fig. 3(d), the space-charge layers are affected by the interaction and also move to the regions most distant from the neighboring streamer.

\section{REFERENCES}

[1] Y. P. Raizer, Gas Discharge Physics. Berlin, Germany: Springer-Verlag, 1991.

[2] K. Takaki, M. Hosokawa, T. Sasaki, S. Mukaigawa, and T. Fujiwara, "Production of atmospheric-pressure glow discharge in nitrogen using needle-array electrode," Appl. Phys. Lett., vol. 86, no. 15, p. 151 501, Apr. 2005.

[3] A. Krasnochub, E. I. Mintoussov, M. Nudnova, and A. Y. Starikovskii, "Interference between streamers in bunch of streamers," in Proc. XXVII Int. Conf. Phenom. Ion. Gases, Veldhoven, The Netherlands, 2005.

[4] E. Gerken, U. Inan, and C. Barrington-Leigh, "Telescopic imaging of sprites," Geophys. Res. Lett., vol. 27, no. 17, pp. 2637-2640, Sep. 2000.

[5] M. McHarg, H. Stenbaek-Nielsen, and T. Kammae, "Observations of streamer formation in sprites," Geophys. Res. Lett., vol. 34, no. 6, p. L06 804, Mar. 2007.

[6] A. Luque, F. Brau, and U. Ebert, Saffman-Taylor Streamer Discharges: A Study on Interacting Streamers, 2008. arXiv:0708.1722. 

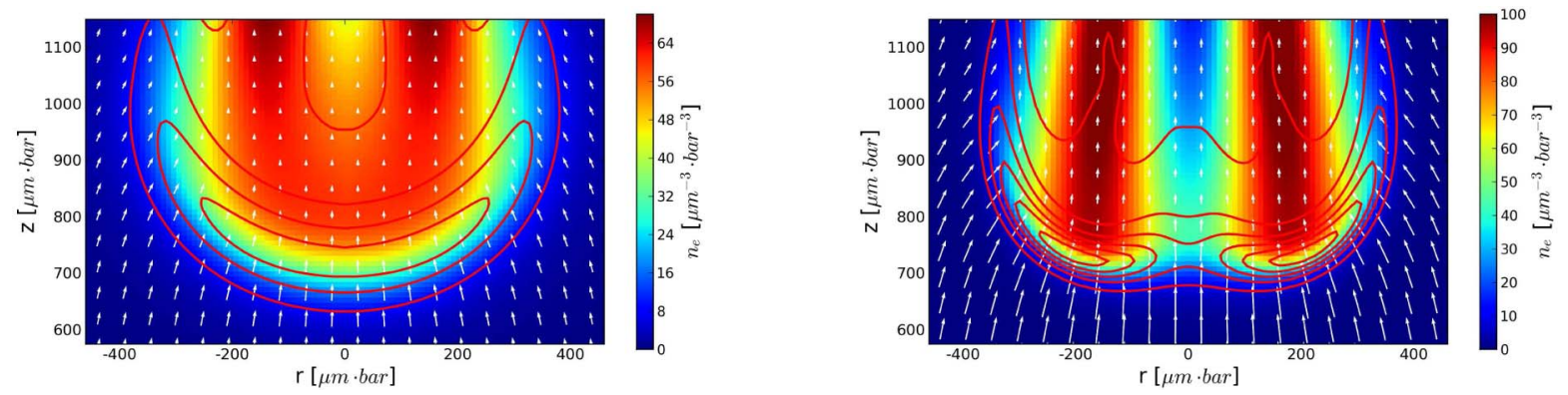

Fig. 1. Electron density (color map), lines of constant charge density (red curves), and electric field (white arrows). (Left) Atmospheric pressure, the streamers coalesce. (Right) Higher pressure (50 bar), the streamers repel, as shown by the obliquous electric field at their heads. Both snapshots were taken at $t=1.7 \mathrm{~ns} \cdot \mathrm{bar}$.

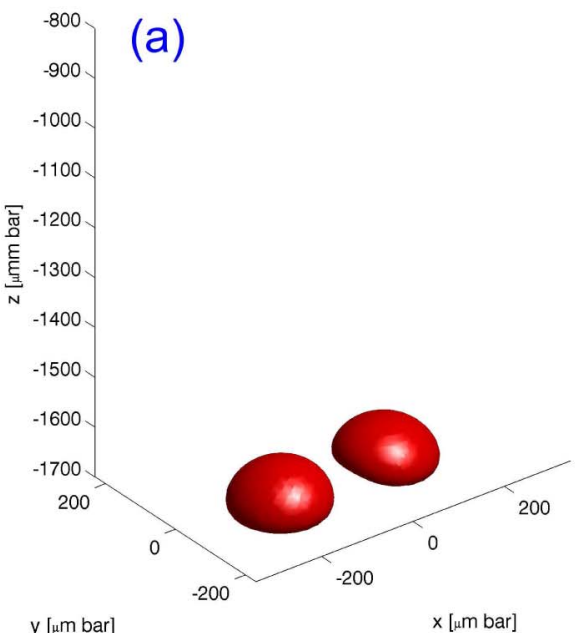

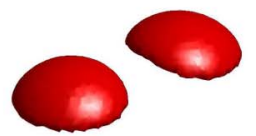
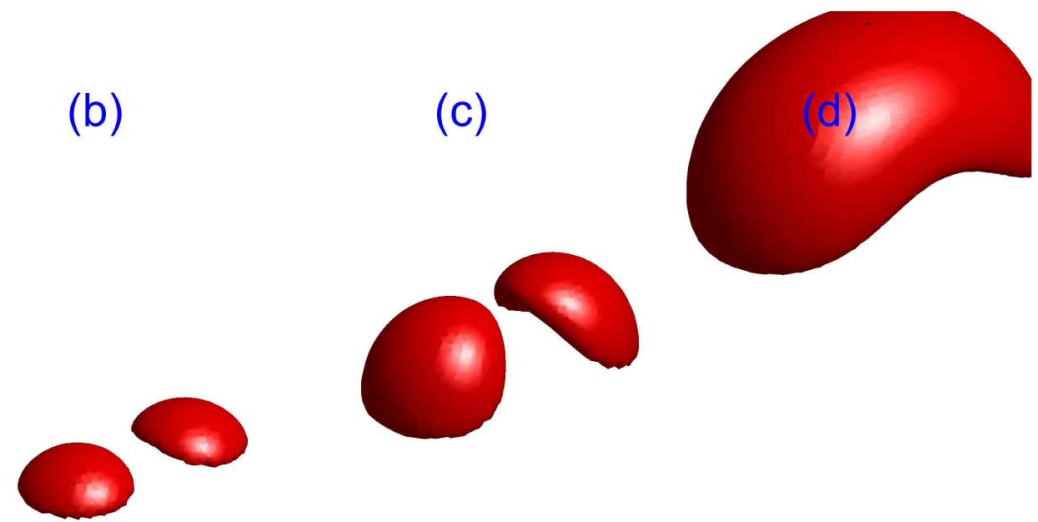

(b)

(c)

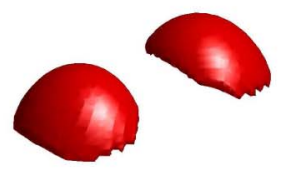

(c)

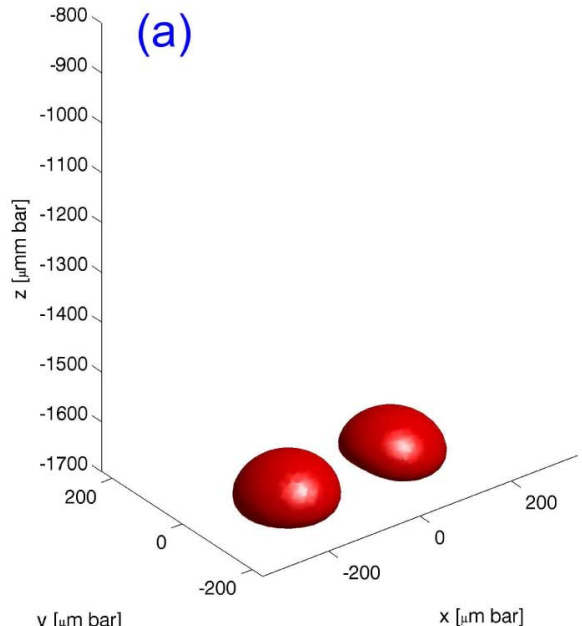

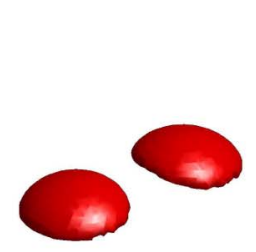

(b) (d)

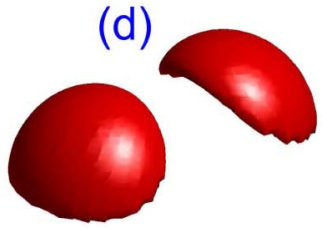

Fig. 3. Same as in Fig. 2 but at 50 bar. In this case, collisional quenching reduces the level of photoionization in the region between the streamers' heads, and hence, they do not merge. Electrostatic repulsion is visible in the outward orientation of the space-charge layers.

[7] A. Luque, U. Ebert, and W. Hundsdorfer, Interaction of Streamers in Air and Other Oxygen-Nitrogen Mixtures, 2008. arXiv:0712.2774.

[8] A. Luque, U. Ebert, C. Montijn, and W. Hundsdorfer, "Photoionization in negative streamers: Fast computations and two propagation modes," Appl. Phys. Lett., vol. 90, no. 8, p. 081 501, Feb. 2007.
[9] J. Wackers, "A nested-grid direct Poisson solver for concentrated source terms," J. Comput. Appl. Math., vol. 180, no. 1, pp. 1-12, Aug. 2005.

[10] C. Montijn, W. Hundsdorfer, and U. Ebert, "An adaptive grid refinement strategy for the simulation of negative streamers," J. Comput. Phys., vol. 219, no. 2, pp. 801-835, Dec. 2006. 